\title{
The correlation between serum AMH and HOMA-IR among PCOS phenotypes
}

\author{
Budi Wiweko*, Indra Indra, Cynthia Susanto, Muharam Natadisastra and Andon Hestiantoro
}

\begin{abstract}
Background: Polycystic ovarian syndrome (PCOS) is known to be one of the most prevalent endocrine disorders affecting reproductive age women. One of the endocrine disorder is hyperinsulinemia, which corresponds with the severity of PCOS. However, the pathogenesis of PCOS is not fully understood, but one theory of anti-mullerian hormone (AMH) has been proposed as one of the factor related to the degree of severity of PCOS. However, there are no clear correlation between levels of AMH with the incidence of insulin resistance in PCOS patients especially in Indonesia.

Methods: This is a cross-sectional study involving reproductive age women aged 18-35 years. Subjects were recruited consecutively at Dr. Cipto Mangunkusumo General Hospital between 2011 until 2014. PCOS women diagnosed using 2003 Rotterdam criteria were categorized into four different PCOS phenotypes. Subsequently, serum level of AMH and HOMA-IR was measured and evaluated with correlation tests performed using SPSS 11.0
\end{abstract}

Results: A total of 125 PCOS patients were included in a study conducted within a 3-year period. Phenotype 1 (anovulation, hyperandrogenism, and polycystic ovaries) shows the highest levels of AMH and HOMA-IR, which decreases in accordance to severity level $(p<0.005)$. The positive correlation between AMH and HOMA-IR persisted even after adjusting for BMI in multivariate analysis.

Conclusions: There was a positive correlation between serum AMH and HOMA IR levels. Serum AMH and HOMA IR levels were significantly different across the four PCOS phenotypes; with the highest values were present with phenotype 1.

Keywords: Anti-mullerian hormone, Insulin resistance, HOMA-IR, Phenotype, Polycystic ovarian syndrome

\section{Background}

Polycystic ovarian syndrome (PCOS) is known to be one of the most prevalent endocrine disorders affecting $15-20 \%$ of reproductive age women and is a primary cause of infertility $[1,2]$. The clinical features of PCOS include menstrual irregularity, chronic anovulation, infertility, and hyperandrogenism [3, 4]. Hormonal imbalance in PCOS manifests as hyperandrogenism and hyperinsulinemia, with reciprocal negative effects and this corresponds with the severity of PCOS. Hyperinsulinemia could increase androgen and free androgen production by reducing the binding of androgen with

*Correspondence: wiwekobudi@yahoo.co.id

Division of Reproductive Endocrinology and Infertility, Department

of Obstetric and Gynecology Faculty of Medicine, Universitas Indonesia;

Dr, Cipto Mangunkusumo General Hospital, Jakarta, Indonesia sex-hormone binding globulin (SHBG). These hormonal imbalances could progress to metabolic and cardiovascular diseases. In a previous cohort study, abnormalities in glucose metabolism were present for $65 \%$ of the cardiovascular disease-related deaths [5].

To date, the pathogenesis of PCOS is not fully understood. One of the proposed mechanism for hyperandrogenism is follicle maturation abnormalities, in which the growing follicle does not progress to a dominant follicle. Follicle maturation depends on the levels of follicle stimulating hormone (FSH), which are reportedly suppressed to a level below the threshold for aromatase enzyme activation in patients with PCOS, resulting in high androgen levels. Another proposed pathology related to follicle maturation abnormality is reduced follicle sensitivity to FSH stimulation by anti-mullerian hormone $(\mathrm{AMH})[6$, 
7]. AMH is a dimeric glycoprotein secreted by the granulose cells, and a member of the transforming growth factor- $\beta$ (TGF- $\beta$ ) family $[8,9]$. In PCOS, levels of AMH are found 3 times higher than in healthy woman [8]. In Indonesia, level of AMH is a useful marker with high sensitivity and specificity for predicting PCOS [10]. The exact mechanism responsible for high level of AMH in PCOS is still poorly understood. Studies concluded that obesity, insulin resistance, and hyperandrogenism play major roles in the increasing level of AMH $[8,11,12]$.

AMH level was recently found to be related to the degree of severity of PCOS $[10,11]$. The severity of PCOS was divided into categories based on patients' phenotypes. According to the Rotterdam criteria (2003) [13], there are four known phenotypes of PCOS, namely: (1) phenotype 1 , characterized by anovulation, hyperandrogenism, and polycystic ovaries; (2) phenotype 2, characterized by anovulation and hyperandrogenism; (3) phenotype 3, characterized by hyperandrogenism and polycystic ovaries; and (4) phenotype 4, characterized by anovulation and polycystic ovaries. Difference in the risks of metabolic disorders were also observed between phenotypes. Similarly, insulin resistance profiles are also found to be different among phenotypes. The most severe form of PCOS is phenotype 1, with higher level of AMH compared to other phenotypes and normal population. However, there are no clear correlations between levels of AMH with the incidence of insulin resistance in PCOS patients. One study involving non-obese Chinese women found $\mathrm{AMH}$ as a useful parameter to assess different phenotypes of PCOS using HOMA-IR marker found no correlation between phenotypes and insulin resistance [14], but similar study has never been conducted on Indonesian population. Thus, the present study tries to look at the relationship between AMH and HOMA-IR in different phenotypes of PCOS especially in the Indonesian population.

\section{Methods}

\section{Study design}

This is a cross-sectional study involving reproductive age women between the age of 18 and 35 years old who came to Yasmin Clinic, Dr. Cipto Mangunkusumo General Hospital, Jakarta, Indonesia between 2011 and 2014. One inclusion criterion is PCOS woman diagnosed using Rotterdam criteria [13], in which diagnosis are established if the patient fulfilled two out of three of the following criteria: (1) oligo-/anovulation, (2) hyperandrogenism (clinically and/or biochemically), (3) polycystic appearance on ultrasound. Another inclusion criterion is serum AMH levels above $4.45 \mathrm{ng} / \mathrm{mL}$. Patients with a history of laparoscopic ovarian drilling and use of insulin sensitizer were excluded from this study.
Ethical approval has been obtained from the Human Ethics Committee of Faculty of Medicine, University of Indonesia. After informed consent was obtained, all patients underwent a general clinical examination.

\section{Measurements and laboratory analyses}

Fasting insulin, AMH and Homeostatic Model Assessment of Insulin Resistance (HOMA-IR) measurements were performed on the serum of each subjects. Level of AMH will be examined using a Gen 2 ELISA kit (Beckman Coulter, Inc. Brea, CA, USA).

Hyperandrogenism is defined as free androgen index (FAI) $>5$; FAI is calculated as testosterone level $(\mathrm{nmol} / \mathrm{L})$ divided by SHBG level [15]. Total testosterone, fasting glucose, fasting insulin, and serum FSH, luteinizing hormone ( $\mathrm{LH})$, and prolactin levels were also examined.

Insulin resistance was measured using the Homeostatic Model Assessment of Insulin Resistance (HOMA-IR) with the following formula: fasting insulin $(\mathrm{mIU} / \mathrm{L}) \times$ fasting plasma glucose $(\mathrm{mg} / \mathrm{dL}) / 405$ [16]

\section{Statistical analysis}

Kolmogorov-Smirnov tests were used to assess the normality of data. Normally distributed numerical data are presented as mean \pm standard deviation, and numerical data not normally distributed are presented as median (range). Kruskal-Wallis test was used to compare the results between the 4 phenotypes; post hoc analyses were conducted using independent $t$ tests or Mann-Whitney tests for normally-distributed and non-normally-distributed data, respectively. Pearson or Spearman correlations were performed with HOMA-IR as the dependent variables. All parameters with a p value $<0.25$ in the correlation analyses were then included in a multivariate linear regression analysis, using a backward elimination method, to determine the variables correlated with the HOMA IR value. Statistical analysis was performed using SPSS for Windows version 11.0 (SPSS Corp, Chicago, IL).

\section{Results}

Subject characteristics

A total of 125 patients were included in this study. The mean age of patients was $29.6 \pm 4.5$ years and the mean BMI was $25.7 \pm 4.8 \mathrm{~kg} / \mathrm{m}^{2}$; with around half of the patients (63 patients, $50.4 \%$ ) were obese (Table 1 ). Phenotype 1 was found to be the most common phenotype among all four phenotypes $(n=39,31.2 \%)$, followed by phenotype 4 (Table 2).

\section{Fasting insulin and plasma glucose levels}

The mean fasting plasma glucose and insulin levels for the entire sample were $103.6 \pm 29.1 \mathrm{mg} / \mathrm{dL}$ and $14.9 \pm 9.6 \mathrm{mIU} / \mathrm{L}$, respectively (Fig. 1). There was a 
Table 1 Characteristics of the subjects with polycystic ovary syndrome

\begin{tabular}{|c|c|c|c|c|}
\hline \multirow[t]{2}{*}{ Characteristics } & \multicolumn{4}{|l|}{ Phenotype } \\
\hline & $\begin{array}{l}1 \\
(n=39)\end{array}$ & $\begin{array}{l}2 \\
(n=27)\end{array}$ & $\begin{array}{l}3 \\
(n=26)\end{array}$ & $\begin{array}{l}4 \\
(n=33)\end{array}$ \\
\hline Age (years) & 29 (20-39) & $29(19-35)$ & $32(23-39)$ & $28(21-36)$ \\
\hline BMI $\left(\mathrm{kg} / \mathrm{m}^{2}\right)$ & $25.5 \pm 4.8$ & $25.8 \pm 3.5$ & $25.7 \pm 5.0$ & $26.0 \pm 5.7$ \\
\hline$\%$ body fat & $31.8(19.2-50.7)$ & $32.1(25.1-45.5)$ & $29.3(23.7-45.8)$ & $34.9(21.1-60.8)$ \\
\hline
\end{tabular}

$\mathrm{BMI}$ are reported as mean \pm standard deviation

Age and \% body fat-values are reported as median (range) since the data distribution is not normal

Table 2 Distribution of the patients among the four polycystic ovarian syndrome phenotypes $(n=125)$

\begin{tabular}{lccccc}
\hline Phenotype & OA & HA & PCO & $\mathbf{n}$ & $\%$ \\
\hline 1 & + & + & + & 39 & 31.2 \\
2 & + & + & - & 27 & 21.6 \\
3 & - & + & + & 26 & 20.8 \\
4 & + & - & + & 33 & 26.4 \\
\hline
\end{tabular}

$O A$ anovulation, $H A$ hyperandrogenism, $P C O$ polycystic ovaries

significant difference in fasting insulin levels among the four phenotypes $(\mathrm{p}=0.014)$ based on the Kruskal-Wallis test.

\section{Hormonal characteristics}

The median AMH level was $8.80(3.10-23.82) \mathrm{ng} / \mathrm{mL}$. Phenotype 1 shows significantly higher AMH levels (Kruskal-Wallis, $\mathrm{p}<0.05$ ) than other phenotypes. Posthoc analyses showed significant differences between phenotypes 1 and 3 , phenotypes 1 and 4, and phenotypes 2 and 4 . Serum LH levels also tended to be higher with phenotype 1. However, there are no differences in FSH level among the four phenotypes. LH levels and LH/FSH ratio were found to be correlated with serum AMH levels $(r=0.58 \mathrm{p}<0.001$ and $r=0.40, \mathrm{p}<0.001$, respectively) (Table 3).

We found HOMA-IR to be correlated with BMI $(r=0.19, \mathrm{p}=0.04)$, LH level $(r=0.27, \mathrm{p}=0.002), \mathrm{LH} /$ FSH ratio $(r=-0.20, \mathrm{p}=0.03)$, and AMH $(r=0.52$, $\mathrm{p}<0.001)$. Thus, BMI, LH/FSH ratio, and AMH were included in the multivariate linear regression analysis (Table 4). The multivariate analysis generated an equation for HOMA IR as follows: $-3.61+0.08(\mathrm{BMI})+5.33(\log$ $\mathrm{AMH}$ ) with an adjusted $\mathrm{R}^{2}=0.30$.

\section{Discussion}

This is the first study looking at the relationship between AMH and HOMA-IR in different types of PCOS phenotypes, especially in Indonesian population. The most prevalent phenotype of PCOS in the present study was phenotype 1 (anovulation, hyperandrogenism, and polycystic ovaries) in which consistent with a previous study showing $46 \%$ of PCOS patients were
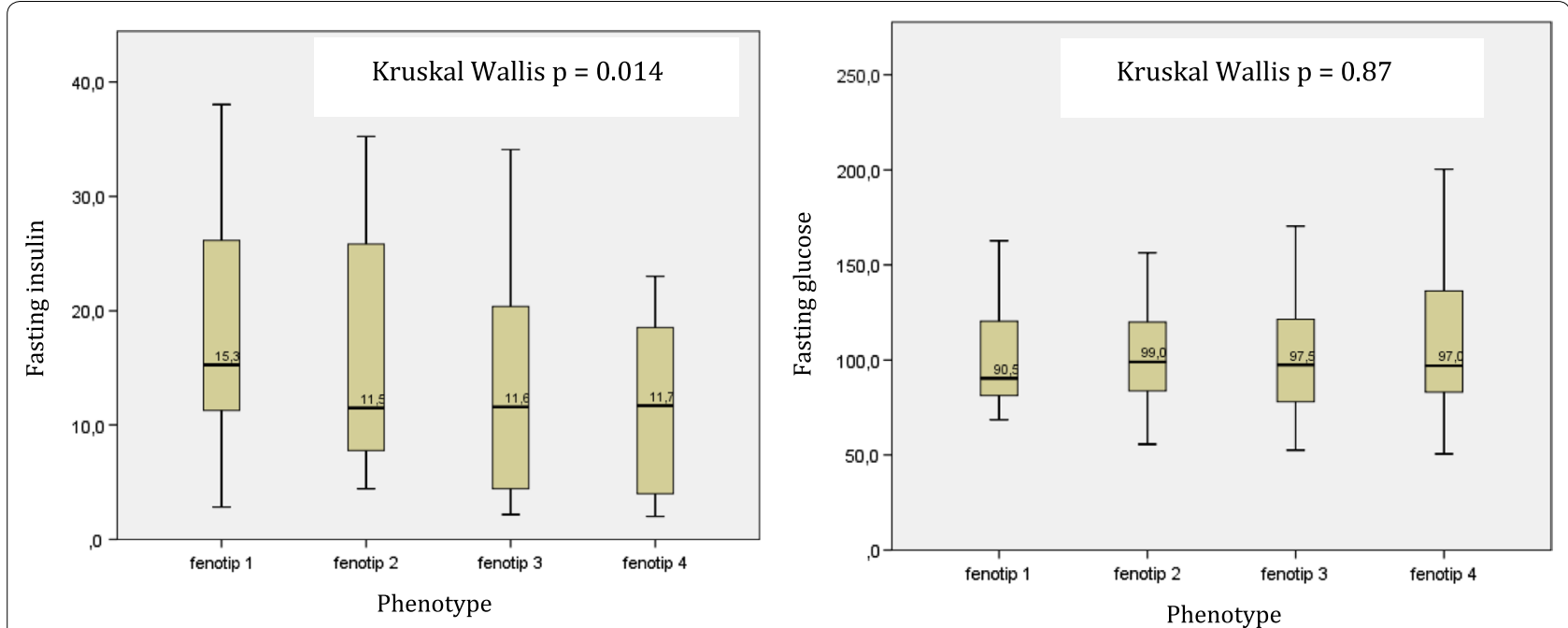

Fig. 1 Fasting serum insulin ( $\mathrm{mlU} / \mathrm{L}$ ) and glucose $(\mathrm{mg} / \mathrm{dL})$ levels in patients with polycystic ovarian syndrome 
Table 3 Hormonal characteristics based on the Rotterdam criteria for polycystic ovarian syndrome

\begin{tabular}{|c|c|c|c|c|}
\hline \multirow{2}{*}{$\begin{array}{l}\text { Hormone } \\
\text { levels }\end{array}$} & \multicolumn{4}{|l|}{ Phenotype } \\
\hline & $\begin{array}{l}1 \\
(n=39)\end{array}$ & $\begin{array}{l}2 \\
(n=27)\end{array}$ & $\begin{array}{l}3 \\
(n=26)\end{array}$ & $\begin{array}{l}4 \\
(n=33)\end{array}$ \\
\hline $\mathrm{AMH}(\mathrm{ng} / \mathrm{mL})$ & $\begin{array}{l}11.7 \\
(4.5-23.8)\end{array}$ & $9.9(4.6-19.5)$ & $7.5(4.6-23.8)$ & $7.1(3.1-9.6)$ \\
\hline $\mathrm{LH}(\mathrm{mlU} / \mathrm{mL})$ & $8.7(5.0-18.8)$ & $8.6(2.0-23.1)$ & $5.1(4.1-13.5)$ & $5.4(2.0-9.9)$ \\
\hline $\begin{array}{c}\mathrm{FSH}(\mathrm{mlU} / \\
\mathrm{mL})\end{array}$ & $4.7(0.4-7.7)$ & $4.0(1.1-6.5)$ & $4.8(0.4-21.2)$ & $5.5(1.7-9.6)$ \\
\hline LH/FSH ratio & $2.1(0.9-32.0)$ & $2.4(0.3-10.6)$ & $1.2(0.5-10.3)$ & $1.1(0.5-4.0)$ \\
\hline HOMA IR & $4.2(0.5-8.2)$ & $3.2(0.7-8.1)$ & $2.9(0.6-8.5)$ & $2.1(0.4-4.9)$ \\
\hline
\end{tabular}

Values are reported as median (range)

Table 4 Linear regression model for the homeostatic model assessment for insulin resistance (HOMA IR)

\begin{tabular}{lccr}
\hline Variables & $\begin{array}{l}\text { Unstandardized B } \\
\text { (standard error) }\end{array}$ & Standardized B & p \\
\hline BMI & $0.08(0.03)$ & 0.19 & 0.015 \\
AMH $^{\text {a }}$ & $5.33(0.76)$ & 0.53 & $<0.001$ \\
Constant & $-3.61(1.09)$ & & 0.001 \\
\hline
\end{tabular}

Adjusted $\mathrm{R}^{2}=0.30$

$B M I$ body mass index, $A M H$ anti-mullerian hormone

${ }^{\text {a }}$ Log-transformed

found to have phenotype 1 [17]. Furthermore, it was found that the more severe the phenotype of PCOS, the higher the level of HOMA-IR. However, this study finds equal distribution among for all four phenotypes, this might be due to the low prevalence of hyperandrogenism in Asian population compared with other races [18, 19].

LH levels and the LH/FSH ratio were highest with phenotype 1 and lowest with phenotype 2, which was similar to the findings of a study conducted in China [20]. A relative FSH deficiency might stimulate the secretion of $\mathrm{LH}$ in patients with PCOS [15]. Moreover, a study conducted in an Iran population revealed high LH levels in phenotype 1 patients corresponding to high levels of both testosterone and androgen [16].

Level of $\mathrm{AMH}$ has also been studied to have correlation with oligo-/anovulation [21] and the level of AMH were 12 times higher among anovulatory PCOS patients with PCOS compared to ovulatory PCOS patients [22].

The mean age of PCOS patients in the present study is 29.6 years old, this is believed to due to a declining number of antral follicles as the patients age advance [23, 24]. However, present study found no significant differences of age group among patients with different PCOS phenotypes.
One of the proposed underlying pathophysiology of PCOS is insulin resistance. It is estimated that around $50-80 \%$ of patients with PCOS also has insulin resistance. In the current study, we found an association between AMH and IR $(r=0.52, \mathrm{p}<0.001)$. Although this relationship remains controversial, with some researchers reporting positive association while others reporting no association, the present study shows very significant correlations between AMH level and IR. Insulin is a reproductive and metabolic hormone. An increase in insulin levels will augment androgen production in PCOS [12].

To date, there is no agreement on which is the best method to evaluate the incidence of insulin resistance in PCOS. Many publications proposed the use of HOMAIR as a standard measurement for insulin resistance diagnosis [25]. The level of HOMA-IR was found at its highest level among PCOS patients with phenotype 1 . The level decreases in accordance with the severity of PCOS. Although BMI did not differ by phenotype, the differences in AMH levels and HOMA-IR tend to follow the spectrum of the four phenotypes. Higher HOMA IR has also been reported in phenotype 1 compared to other phenotypes as well as controls [20]. Fasting insulin level significantly differs between all phenotypes (Fig. 1), however, consistent with a previous study, we found no difference in fasting plasma glucose levels [16]. Thus, patients with PCOS might have abnormal insulin levels despite having normal plasma glucose levels. Although current guidelines focus on plasma glucose to diagnose diabetes in the general population [26], physicians treating patients with PCOS should not rely only on normal glucose levels. Moreover, these findings might support the benefit of metformin therapy to lower androgen levels in patients with PCOS [27].

A significant positive correlation between $\mathrm{AMH}$ and HOMA-IR was observed in the current study, despite the fact that a causative relationship could not be determined. This possible link between the two hormones has been discussed among healthy women population [28]. Skalba, et al. found a moderate positive correlation between AMH and HOMA-IR $(r=0.62$, p < 0.001) [29]. However, a negative correlation between $\mathrm{AMH}$ and HOMA-IR has also been reported [9].

This study has several limitations. Owing to its crosssectional design, a causative relationship between AMH and insulin resistance could not be explored. Data regarding standardized screening of diabetes is also limited, and previous history of diabetes mellitus were not obtained. Furthermore, because patients were recruited from a tertiary hospital, previous medication history could not be fully obtained. Additionally, other variables related to insulin resistance (e.g., physical activity and diet history) could not be quantified. The correlation between the 
AMH and HOMA-IR values should be interpreted with caution, as AMH might be a predictor of insulin resistance. To our knowledge, no study has reported direct relationship between AMH and insulin resistance. Due to a lack of data regarding androgen levels, the results might be overestimate as AMH is also reported to be positively associated with serum androgen levels [30].

\section{Conclusions}

There was a positive correlation between serum AMH and HOMA-IR levels. Serum AMH and HOMA-IR levels were significantly different across the four PCOS phenotypes; with the highest values observed in patients with phenotype 1.

\section{Abbreviations \\ PCOS: polycystic ovarian syndrome; AMH: anti-mullerian hormone; HOMA-IR: homeostatic model assessment for insulin resistance; SHBG: sex hormone binding globulin; BMI: body mass index; FSH: follicle stimulating hormone; LH: luteinizing hormone; ELISA: enzyme linked immunoabsorbent assay; TGF- $\beta$ : transforming growth factor $\beta$.}

\section{Authors' contributions}

BW conceived of the study, carried out the design study, recruited the subject, drafted and finalized the manuscript, II participated in laboratory analysis and performed statistical analysis, CS participated in sample collection, drafted and edited the manuscript. MN participated in the recruitment of the subject and data collection, $\mathrm{AH}$ participated in carried out the study and drafted the manuscript. All authors read and approved the final manuscript.

\section{Acknowledgements}

The authors would like to thank Gita Pratama, Achmad Kemal, Mila Maidarti, Kresna Mutia, Pritta Amelia, Dwinarsi Yusuf and all of the clinicians and staff of the Division of Reproductive Endocrinology and Infertility, Department of Obstetrics and Gynecology, Faculty of Medicine Universitas Indonesia.

\section{Competing of interests}

The authors declare that they have no competing interests.

\section{Availability of data and materials}

The datasets used and/or analyzed during the current study are available from the corresponding author on reasonable request.

\section{Consent for publication}

There are no details images or videos relation to an individual person upon this study.

\section{Ethics approval and consent to participate}

Ethical approval has been obtained from the Human Ethics Committee of Faculty of Medicine, University of Indonesia. Written consent was obtained from each of the subject before subject can be included in the study.

\section{Funding}

The funding for this study was not applicable.

\section{Publisher's Note}

Springer Nature remains neutral with regard to jurisdictional claims in published maps and institutional affiliations.

Received: 14 March 2016 Accepted: 25 January 2018

Published online: 09 February 2018
References

1. Sirman S, Pate KA. Epidemiology, diagnosis, and management of polycystic ovary syndrome. Clin Epidemiol. 2014;6:1-13.

2. Dunaif A. Insulin resistance and the polycystic ovary syndrome: mechanism and implications for pathogenesis. Endocr Rev. 1997;18:774-800.

3. Jakubowicz DJ, luorno MJ, Jakubowicz S, Roberts KA, Nestler JE. Effects of metformin on early pregnancy loss in the polycystic ovary syndrome. J Clin Endocrinol Metab. 2002;87:524-9.

4. Hopkinson ZE, Sattar N, Fleming R, Greer IA. Polycystic ovarian syndrome: the metabolic syndrome comes to gynaecology. BMJ. 1998;317:329-32.

5. Teede H, Deeks A, Moran L. Polycystic ovary syndrome: a complex condition with psychological, reproductive and metabolic manifestations that impacts on health across the lifespan. BMC Med. 2010;8:41.

6. Wachs DS, Coffler MS, Malcom PJ, Chang RJ. Serum anti-mullerian hormone concentrations are not altered by acute administration of follicle stimulating hormone in polycystic ovary syndrome and normal women. J Clin Endocrinol Metab. 2007;92:1871-4.

7. La Marca A, Broekmans FJ, Volpe A, Fauser BC, Macklon NS, ESHRE Special Interest Group for Reproductive Endocrinology-AMH Round Table. Anti-müllerian hormone (AMH): what do we still need to know? Hum Reprod. 2009;24:2264-75.

8. Pellatt L, Rice $S$, Mason HD. Anti-müllerian hormone and polycystic ovary syndrome: a mountain too high? Reproduction. 2010;139:825-33.

9. Chen MJ, Yang WS, Chen CL, Wu MY, Yang YS, Ho HN. The relationship between anti-mullerian hormone, androgen and insulin resistance on the number of antral follicles in women with polycystic ovary syndrome. Hum Reprod. 2008:23:952-7.

10. Wiweko B, Maidarti M, Priangga MD, Shafira N, Fernando D, Sumapraja K, Natadisastra M, Hestiantoro A. Anti-mullerian hormone as a diagnostic and prognostic tool for PCOS patients. J Assist Reprod Genet. 2014:31:1311-6.

11. Piouka A, Farmakiotis D, Katsikis I, Macut D, Gerou S, Panidis D. Antimullerian hormone levels reflect severity of PCOS but are negatively influenced by obesity: relationship with increased luteinizing hormone levels. Am J Physiol Endocrinol Metab. 2009;296:E238-43.

12. Diamanti-Kandarakis $E$, Dunaif $A$. Insulin resistance and the polycystic ovary syndrome revisited: an update on mechanism and implications. Endocr Rev. 2012;33(6):981-1030.

13. Rotterdam ES, ASRM-Sponsored PCOS Consensus Workshop Group. Revised 2003 consensus on diagnostic criteria and long-term health risks related to polycystic ovary syndrome (PCOS). Hum Reprod. 2004;19(1):41-7 (Epub 2003/12/23).

14. Tian X, Ruan X, Mueck AO, Wallwiener D, Wang J, et al. Serum anti-mullerian hormone and insulin resistance in the main phenotypes of nonobese polycystic ovarian syndrome women in China. Gynecol Endocrinol. 2014;30(11):836-9.

15. Louwers YV. Polycystic ovary syndrome: from phenotype to genotype. Rotterdam: Thesis, Erasmus University Rotterdam; 2014.

16. Tehrani FR, Rashidi H, Khomami MB, Tohidi M, Azizi F. The prevalence of metabolic disorders in various phenotypes of polycystic ovary syndrome: a community based study in Southwest of Iran. Reprod Biol Endocrinol. $2014 ; 12: 89$

17. Diamanti-Kandarakis E, Panidis D. Unravelling the phenotypic map of polycystic ovary syndrome (PCOS): a prospective study of 634 women with PCOS. Clin Endocrinol (Oxf). 2007;67:735-42.

18. Li L, Chen X, He Z, Zhao X, Huang L, Yang D. Clinical and metabolic features of polycystic ovary syndrome among Chinese adolescents. J Pediatr Adolesc Gynecol. 2012;25:390-5.

19. Tehrani FR, Rashidi H, Khomami MB, Tohidi M, Azizi F. The prevalence of metabolic disorders in various phenotypes of polycystic ovary syndrome: a community based study in Southwest of Iran. Reprod Biol Endocrinol. 2014;12:89.

20. Zhang HY, Zhu FF, Xiong J, Shi XB, Fu SX. Characteristics of different phenotypes of polycystic ovary syndrome based on the Rotterdam criteria in a large-scale Chinese population. BJOG. 2009;116:1633-9.

21. Zawadski J, Dunaif A. Diagnostic criteria for polycystic ovary syndrome: towards a rational approach. In: Dunaif A, Givens J, Haseltine F, Merriam G, Hershman S, editors. Polycystic ovary syndrome current issues in endocrinology and metabolism. Boston: Blackwell Scientific Publications; 1992. 
22. Caglar GS, Kahyaoglu I, Pabuccu R, Demirtas S, Seker R. Anti-Mullerian hormone and insulin resistance in classic phenotype lean PCOS. Arch Gynecol Obstet. 2013;288:905-10.

23. Johnstone EB, Rousseau JA, Lamb JD, Huddleston HG, Cedars MI. Age bias in polycystic ovary syndrome (PCOS) diagnostic criteria limits diagnosis among those at greatest cardiovascular risk. Fertil Steril. 2009;92:S38.

24. Murphy MK, Hall JE, Adams JM, Lee H, Welt CK. Polycystic ovarian morphology in normal women does not predict the development of polycystic ovary syndrome. J Clin Endocrinol Metab. 2006:91:3878-84.

25. Gayoso-Diz P, Otero-González A, Rodriguez-Alvarez MX, Gude F, García F, De Francisco A, Quintela AG. Insulin resistance (HOMA-IR) cut-off values and the metabolic syndrome in a general adult population: effect of gender and age: EPIRCE cross-sectional study. BMC Endocr Disord. 2013;13:47.

26. Sacks DB, Arnold M, Bakris GL, Bruns DE, Horvath AR, Kirkman MS, Lernmark A, Metzger BE, Nathan DM, National Academy of Clinical Biochemistry. Guidelines and recommendations for laboratory analysis in the diagnosis and management of diabetes mellitus. Diabetes Care. 2011;34:e61-99.

27. Speroff L, Fritz MA. Clinical gynecologic endocrinology and infertility. 7th ed. Philadephia: Lippincot Williams and Wilkins; 2005.

28. Park HT, Cho GJ, Ahn KH, Shin JH, Kim YT, Hur JY, Kim SH, Lee KW, Kim T. Association of insulin resistance with anti-mullerian hormone levels in women without polycystic ovary syndrome (PCOS). Clin Endocrinol (Oxf) 2010;72:26-31.

29. Skalba P, Cygal A, Madej P, Dabkowska-Huc A, Sikora J, Martirosian G, Romanik M, Olszanecka-Glinianowicz M. Is the plasma anti-müllerian hormone (AMH) level associated with body weight and metabolic, and hormonal disturbances in women with and without polycystic ovary syndrome? Eur J Obstet Gynecol Reprod Biol. 2011;158:254-9.

30. Eldar-Geva T, Margalioth EJ, Gal M, Ben-Chetrit A, Algur N, Zylber-Haran E, Brooks B, Huerta M, Spitz M. Serum anti-mullerian hormone levels during controlled ovarian hyperstimulation in women with polycystic ovaries with and without hyperandrogenism. Hum Reprod. 2005;20:1814-9.

\section{Submit your next manuscript to BioMed Central and we will help you at every step:}

- We accept pre-submission inquiries

- Our selector tool helps you to find the most relevant journal

- We provide round the clock customer support

- Convenient online submission

- Thorough peer review

- Inclusion in PubMed and all major indexing services

- Maximum visibility for your research

Submit your manuscript at www.biomedcentral com/submit 